\title{
Probing nuclear symmetry energy at high densities using pion, kaon, eta and photon productions in heavy-ion collisions
}

\author{
Zhi-Gang Xiao ${ }^{1,2}$, Gao-Chan Yong ${ }^{3}$, Lie-Wen Chen ${ }^{4}$, Bao-An Li ${ }^{5}$, Ming Zhang ${ }^{6}$, Guo-Qing Xiao ${ }^{3}$, and Nu Xu \\ 1 Department of Physics, Tsinghua University, Beijing 100084, China \\ 2 Collaborative Innovation Center of Quantum Matter, Beijing, China \\ 3 Institute of Modern Physics, Chinese Academy of Sciences, Lanzhou 730000, China \\ 4 Department of Physics and Astronomy, Shanghai Jiao Tong University, Shanghai 200240, China \\ 5 Department of Physics and Astronomy, Texas A\&M University-Commerce, Commerce, Texas 75429-3011, USA \\ 6 National Institute of Metrology, Beijing 100013, China \\ 7 Institute of Particle Physics, Central China Normal University, Wuhan 430079, China
}

Received: date / Revised version: date

\begin{abstract}
The high-density behavior of nuclear symmetry energy is among the most uncertain properties of dense neutron-rich matter. Its accurate determination has significant ramifications in understanding not only the reaction dynamics of heavy-ion reactions especially those induced by radioactive beams but also many interesting phenomena in astrophysics, such as the explosion mechanism of supernova and the properties of neutron stars. The heavy-ion physics community has devoted much effort during the last few years to constrain the high-density symmetry using various probes. In particular, the $\pi^{-} / \pi^{+}$ratio has been most extensively studied both theoretically and experimentally. All models have consistently predicted qualitatively that the $\pi^{-} / \pi^{+}$ratio is a sensitive probe of the high-density symmetry energy especially with beam energies near the pion production threshold. However, the predicted values of the $\pi^{-} / \pi^{+}$ratio are still quite model dependent mostly because of the complexity of modeling pion production and reabsorption dynamics in heavy-ion collisions, leading to currently still controversial conclusions regarding the highdensity behavior of nuclear symmetry energy from comparing various model calculations with available experimental data. As more $\pi^{-} / \pi^{+}$data become available and a deeper understanding about the pion dynamics in heavy-ion reactions is obtained, more penetrating probes, such as the $K^{+} / K^{0}$ ratio, $\eta$ meson and high energy photons are also being investigated or planned at several facilities. Here, we review some of our recent contributions to the community effort of constraining the high-density behavior of nuclear symmetry energy in heavy-ion collisions. In addition, the status of some worldwide experiments for studying the high-density symmetry energy, including the HIRFL-CSR external target experiment (CEE) are briefly introduced.
\end{abstract}

PACS. symmetry energy, supra-saturation density

\section{Introduction}

Over a very wide beam energy range from tens of $\mathrm{MeV} / \mathrm{u}$ to several $\mathrm{TeV} / \mathrm{u}$, one of the main purposes of heavy-ion collisions is to study the bulk properties of strong interaction matter and understand the QCD phase diagram. In the hardonic phase, the symmetry energy term $E_{\mathrm{sym}}(\rho)$ in the equation of state (EOS) of isospin-asymmetric nuclear matter (with unequal fractions of neutrons and protons) is presently subject to the largest uncertainty among all properties of nucleonic matter. The density dependence of nuclear symmetry energy is an essential input for calculating the properties of neutron stars. Moreover, it is also important for understanding nuclear structure and reactions, particularly those involving exotic nuclei [1,2]. While some significant progress has been made over the last few years in constraining the $E_{\text {sym }}(\rho)$ at sub-saturation densities using both terrestrial nuclear laboratory data and astrophysical observations 3, 4, 5, 6, still very little is known about the $E_{\text {sym }}(\rho)$ at supra-saturation densities.

Thanks to the collaborative effort of the community during the last decade, several promising probes of the symmetry energy at supra-saturation densities have been identified, such as the $\pi^{-} / \pi^{+}$[7,8, and $K^{+} / K^{0}[8,9$ ratios, the neutron-proton differential transverse [10] and elliptic flows [11, the neutron/proton ratio of squeezed-out nucleons [12, triton over ${ }^{3} \mathrm{He}$ ratio [13 14, $\Sigma^{-} / \Sigma^{+}$ratio 15], $\eta$ meson [16] and high energy photon [17,18, productions. However, presently our conclusions regarding the highdensity behavior of nuclear symmetry energy are still inconclusive, and in some cases controversial. For example, the FOPI collaboration at GSI, Darmstadt published a 
few years ago a complete set of pion data in heavy-ion collisions [19. From analyzing the charged pion ratio within the IBUU04 transport model [20, a circumstantial evidence for a super-soft symmetry energy was reported 21. However, an analysis of the same data using a version of the quantum molecular dynamics model gave an opposite conclusion 23. More recent studies using the BoltzmannLangiven approach 24 made a similar conclusion as in ref. [21]. This situation has stimulated some interesting debate and certainly calls for more studies, such as, the inmedium effects of $\Delta$ and pion production 25, 26, 27, both experimentally and theoretically. It is interesting to note that many theoretical studies have been performed to better understand the causes of the uncertain symmetry energy at supra-saturation densities. In particular, effects of the three-body force 28, 29, isospin dependence of shortrange nucleon-nucleon correlation and tensor force 30,31, high order isospin asymmetry effect 32,33 and exchange of $\rho-\omega$ mesons [34, deserve special attention. In this article, we highlight a few key points reported in several of our recent studies about probing the high-density symmetry energy using pion, kaon, eta and high energy photon production in heavy-ion collisions. Several newly planned experiments are also discussed.

The paper is arranged as following. Section 2 explains why heavy ion collisions in terrestrial laboratory are useful for determining $E_{\text {sym }}(\rho)$. Section 3 discusses the pion, kaon, $\eta$ and photon productions and their relevance in constraining $E_{\text {sym }}(\rho)$ at supra-saturation densities. Section 4 introduces briefly the future experiments planned worldwide and Section 5 is the summary.

\section{Symmetry energy and the isospin asymmetry of dense matter}

Why are the heavy-ion collisions useful for probing the symmetry energy at supra-saturation densities? It is necessary to first answer this question before we discuss potential probes of the high-density symmetry energy in heavy-ion collisions. The answer lies in the fact that the isospin asymmetry of dense matter formed in heavy-ion collisions are intimately related to the density dependence of nuclear symmetry energy. As an example, shown in the upper window of Fig. 1 are two possible functions of the symmetry energy $E_{\text {sym }}(\rho)$. As discussed earlier in Refs. 10, 35, the considered two forms of the symmetry energy have the same value of $E_{\text {sym }}\left(\rho_{0}\right)=30 \mathrm{MeV}$ at the normal nuclear matter density $\rho_{0}$ and are very close to each other at lower densities. At high densities they have completely different trends reflecting the diverging predictions of nuclear many-body theories. In the simplest model of neutron stars consisting of npe matter at $\beta$ equilibrium, the proton fraction $x_{\beta}$ entirely determined by the $E_{\mathrm{sym}}(\rho)$ is shown in the lower window of Fig. 1. With the $E_{\mathrm{sym}}^{b}(\rho)$, the $x_{\beta}$ is zero for $\rho / \rho_{0} \geq 3$, while with the $E_{\text {sym }}^{a}(\rho)$, the neutron star becomes so proton-rich that the fast cooling can happen at densities higher than about $2.3 \rho_{0}$.

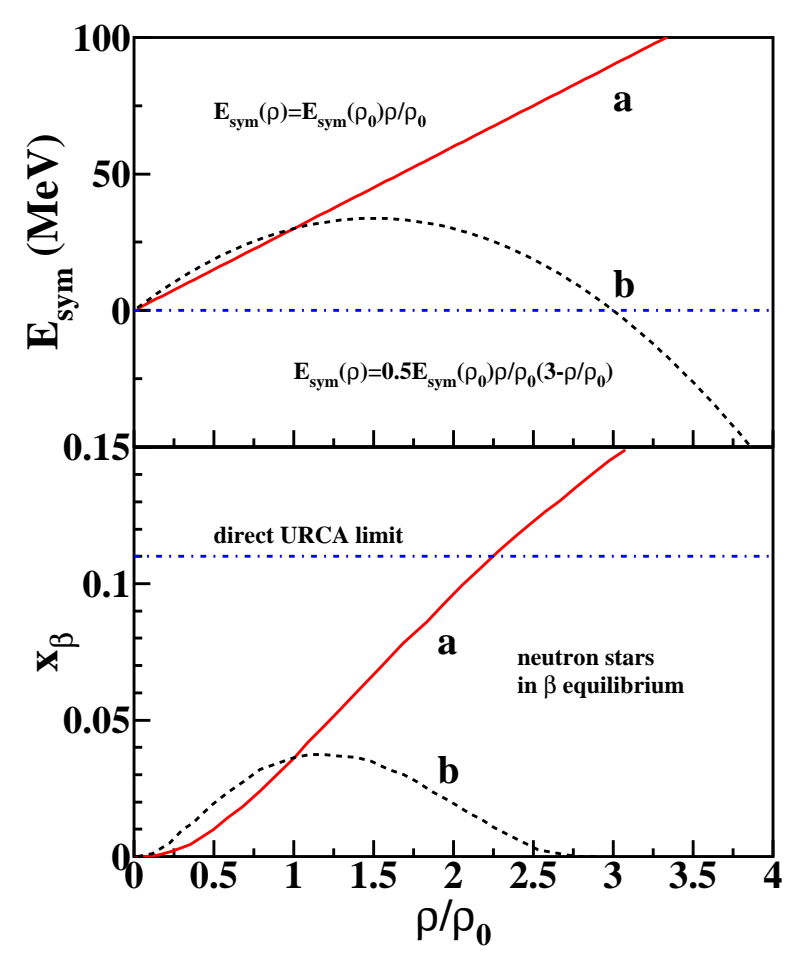

Fig. 1. (color online) Upper window: Two representatives of the nuclear symmetry energy as a function of density. Lower window: the corresponding proton fractions in neutron stars at $\beta$ equilibrium. Taken from Refs. 10,35.

Similar to the isospin fractionation in liquid-gas phase transition in heavy-ion collisions at intermediate energies [36, 37, 38, the different high-density behaviors of the symmetry energy also lead to very different isospin asymmetry in heavy-ion collisions. As examples, shown in Fig. 2 are the correlations between the baryon density and the isospin asymmetry $\delta_{\text {like }}$ over the entire reaction volume at the time of about the maximum compression in the central ${ }^{132} \mathrm{Sn}+{ }^{124} \mathrm{Sn}$ reactions with beam energy of 400 (upper window) and 2000 (bottom window) $\mathrm{MeV} / \mathrm{u}$, respectively. How the initial $\rho-\delta$ correlation evolves in a heavy ion collision depends sensitively on the high-density behavior of nuclear symmetry energy. With the $E_{\text {sym }}^{a}(\rho)$ the continuous neutron distillation from higher density regions to lower ones persists at all energies. For instance,the isospin asymmetry of the high-density region around $\rho=2 \rho_{0}$ with $E_{\mathrm{sym}}^{b}(\rho)$ is about twice that with the $E_{\mathrm{sym}}^{a}(\rho)$ at 400 $\mathrm{MeV} / \mathrm{u}$. It is very interesting to note the astonishing similarity in the resultant $\delta-\rho$ correlations for neutron stars and heavy-ion collisions. In both cases, the symmetry energy $E_{\text {sym }}^{b}(\rho)$ makes the high-density nuclear matter more neutron-rich than the $E_{\mathrm{sym}}^{a}(\rho)$ and the effect grows with the increasing density [10,35. 


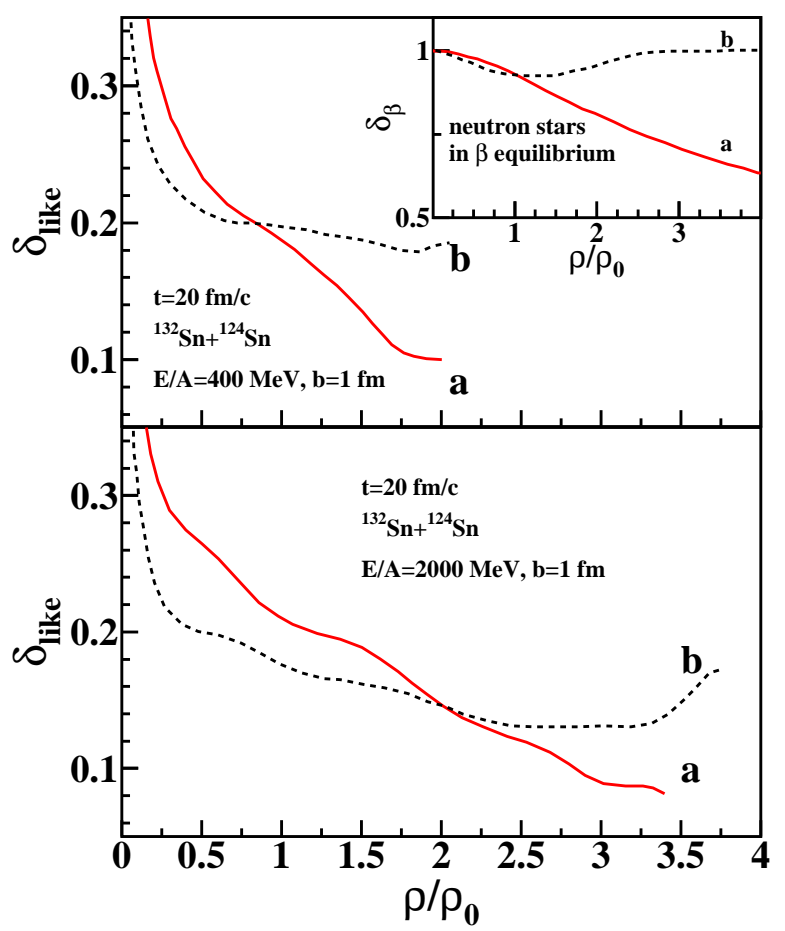

Fig. 2. (color online) Upper window: the isospin asymmetrydensity correlations at $\mathrm{t}=20 \mathrm{fm} / \mathrm{c}$ and $E_{\text {beam }}=400 \mathrm{MeV} / \mathrm{u}$ in the central ${ }^{132} \mathrm{Sn}+{ }^{124} \mathrm{Sn}$ reaction with the nuclear symmetry energy $E_{\text {sym }}^{a}$ and $E_{\text {sym }}^{b}$, respectively. Lower window: the same correlation as in the upper window but at $10 \mathrm{fm} / \mathrm{c}$ and $E_{\text {beam }}=$ $2 \mathrm{GeV} / \mathrm{u}$. The corresponding correlation in neutron stars is shown in the insert. Taken from Refs. 10, 35.

\section{Particle production in heavy-ion collisions as potential probes of the symmetry energy at supra-saturation densities}

In the following we review a few recent studies about using pion, kaon and $\eta$ mesons as well as high energy photons as potential probes of the high-density symmetry energy.

\subsection{The $\pi^{-} / \pi^{+}$ratio as a probe of nuclear symmetry energy}

As discussed in detail in the literature, see, e.g., Refs. [10, 35, 39, 40, 24, 42, the $\pi^{-} / \pi^{+}$ratio in heavy-ion collisions may be used as a sensitive probe of the high-density behavior of nuclear symmetry energy. In fact, it was realized a long time ago that the $\pi^{-} / \pi^{+}$ratio depends strongly on the isospin asymmetry of the reaction system, see, e.g., Refs [41,43, 44,45. This can be understood qualitatively from both the $\Delta$ resonance model and the statistical model for pion production. Within the $\Delta$ resonance model for pion production from first-chance independent nucleon-nucleon collisions [45, the primordial $\pi^{-} / \pi^{+}$ratio is $\left(5 N^{2}+N Z\right) /\left(5 Z^{2}+N Z\right) \approx(N / Z)^{2}$. It is thus a direct measure of the isospin asymmetry $(N / Z)_{\text {dense }}$ of the dense matter in the participant region of heavy-ion collisions. As we have discussed earlier, the $(N / Z)_{\text {dense }}$ is uniquely determined by the high-density behavior of the nuclear symmetry energy. Therefore, the $\pi^{-} / \pi^{+}$ratio can be used to probe sensitively the high-density behavior of nuclear symmetry energy. On the other hand, within the statistical model for pion production [46], the $\pi^{-} / \pi^{+}$ratio is proportional to $\exp \left[\left(\mu_{n}-\mu_{p}\right) / T\right]$, where $T$ is the temperature, $\mu_{n}$ and $\mu_{p}$ are the chemical potentials of neutrons and protons, respectively. At modestly high temperatures $(T \geq 4 \mathrm{MeV})$, the difference in the neutron and proton chemical potentials can be written as 47]

$$
\begin{aligned}
\mu_{n}-\mu_{p} & =V_{a s y}^{n}-V_{a s y}^{p}-V_{\text {Coulomb }} \\
& +T\left[\ln \frac{\rho_{n}}{\rho_{p}}+\sum_{m} \frac{m+1}{m} b_{m}\left(\frac{\lambda_{T}^{3}}{2}\right)^{m}\left(\rho_{n}^{m}-\rho_{p}^{m}\right)\right]
\end{aligned}
$$

where $V_{\text {Coulomb }}$ is the Coulomb potential for protons, $\lambda_{T}$ is the thermal wavelength of a nucleon and $b_{m}^{\prime} \mathrm{s}$ are the inversion coefficients of the Fermi distribution function 47]. The difference in neutron and proton potentials $V_{a s y}^{n}-V_{a s y}^{p}=$ $2 v_{a s y}(\rho) \delta$, where the symmetry potential $v_{a s y}(\rho)$ is directly related to the symmetry energy $48,49,50$. It is seen that the difference $\mu_{n}-\mu_{p}$ relates directly to the isospin asymmetry $\rho_{n} / \rho_{p}$ or $\rho_{n}-\rho_{p}$. Thus within the statistical model, the $\pi^{-} / \pi^{+}$ratio is also sensitive to the $(N / Z)_{\text {dense. }}$ Moreover, the value of $\pi^{-} / \pi^{+}$ratio is affected by the competition of the symmetry and Coulomb potentials which all depend on the isospin asymmetry of the reaction system. While the expectations based on these two idealized models provide a useful guide, advanced transport model calculations are necessary. Indeed, extensive studies have been done using various versions of transport models for haevy-ion collisions. One such model is the isospin- and momentum- dependent Boltzmann-UehlingUhlenbeck transport model (IBUU04). In this model, the isospin-dependent in-medium nucleon-nucleon (NN) elastic scattering cross sections are adopted, while the inelastic cross sections are extracted from experimental freespace NN inelastic collisions. For the single nucleon potential, the modified Gogny momentum dependent interaction (MDI) is used. With this potential the neutron effective mass is higher than the proton effective mass in neutron-rich nuclear matter and the splitting between them increases with both the density and isospin asymmetry of the medium. By integrating over the momentum, the symmetry energy as a function of density is then derived and plotted in Fig. 3 . The variable $x$ is introduced to mimic different forms of the $E_{\mathrm{sym}}(\rho)$ predicted by various many body theories without changing any property of the symmetric nuclear matter and the symmetry energy at normal density [51. For comparison, the well-known Akmal-Pandharipande-Ravenhall (APR) prediction [52] (blue asterisks) and the parametrization used in the Isospin-dependent Quantum Molecular Dynamics (IQMD) model [53] (pink dotted) are also presented.

Comparisons of the FOPI data and the IBUU04 calculations are shown in Fig. 4. The window (a) displays the 


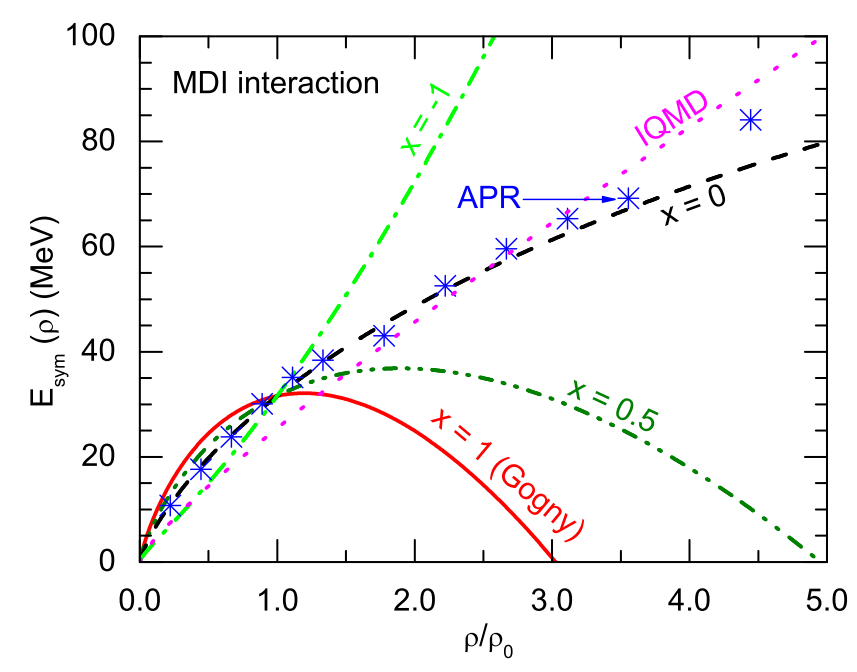

Fig. 3. (Color Online) The symmetry energy as a function of densities derived from the modified Gogny momentum dependent interaction. The well-known APR prediction 52 (blue asterisks) and the parametrization used in IQMD model 53 (pink dotted) are also plotted for comparison. Taken from ref. 21.

reduced multiplicity of the charged pions $M_{\pi} / A_{\text {part }}$ where $A_{\text {part }}$ denotes the number of participant nucleons. It is seen that the total pion yield exhibits insignificant dependence on the symmetry energy parameter $x$. However, if we inspect the pion yield ratios $\pi^{-} / \pi^{+}$as a function of beam energy (b) and of system isospin composition (c), it is interesting to notice that the FOPI data favors clearly the calculation with $\mathrm{x}=1$ corresponding to a very soft symmetry energy. The calculation with $x=0$ is consistent with the IQMD calculations but deviates considerably from the data [21. As discussed above, an enhanced pion ratio $\pi^{-} / \pi^{+}$means that more neutrons are resided in the high-density region to produce more $\pi^{-}$according to the simple isobar model. To achieve a neutron rich dense region, a soft symmetry energy is required. It is worth noting that the super-soft symmetry energy can not support massive neutron stars with the normal EOS. This has triggered the interesting application of non-Newtonian gravity in neutron stars [54]. On the other hand we would also note that the symmetry energy sensitivity of the $\pi^{-} / \pi^{+}$ratio only shows up below $600 \mathrm{MeV} / \mathrm{u}$ corresponding to a nuclear matter density approximately lower than $2 \rho_{0}$.

It should be pointed out that model dependent conclusions have been reported based on the same data set from FOPI collaboration. Using an isospin dependent quantum molecular dynamics (IQMD) model, Feng et al. have found that in order to reproduce the data a rather stiff symmetry energy is necessary [23. In another analysis done recently by Xie et al. using the Boltzmann-Langevin equations, a very soft symmetry energy similar with the curve represented by $x=1$ in IBUU04 analysis is favored [24.

To arrive at a convincing constraint of the symmetry energy at supra-saturation densities from the pionic probe, more theoretical and experimental studies are required.

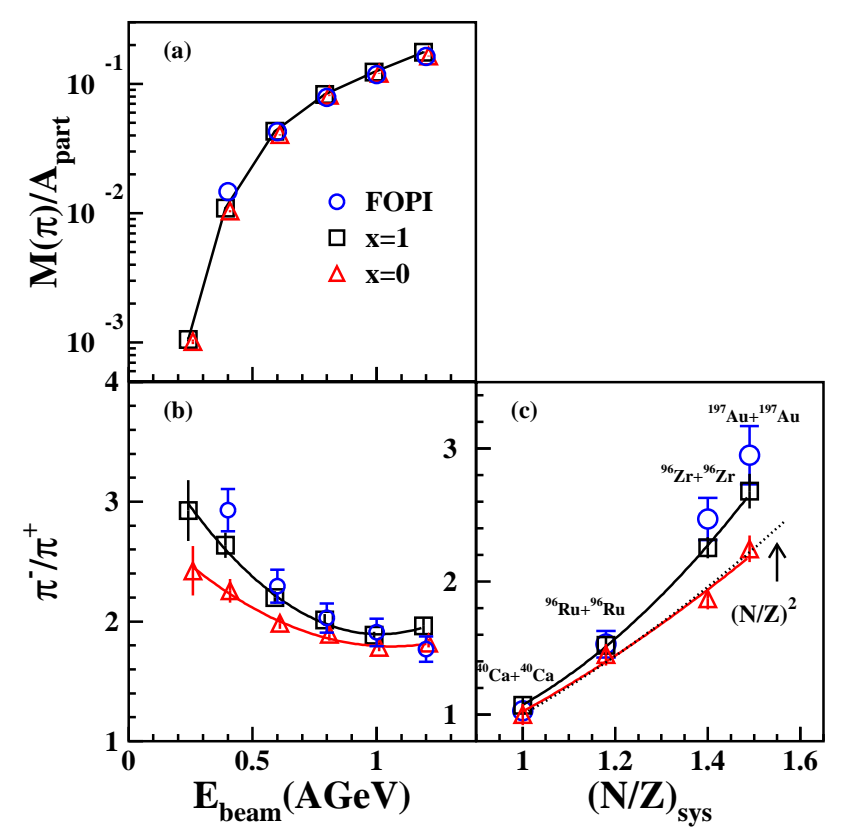

Fig. 4. (Color Online) The excitation function of the total yield of charged pions (a) and of the yield ratio $\pi^{-} / \pi^{+}$(b). (c) The yield ratio $\pi^{-} / \pi^{+}$as a function of the system $N / Z$. Taken from ref. [21,22].

From experimental point of view, it is of significance to optimize the conditions such as the reaction system and the beam energies to achieve the maximum sensitivity that the probes depend on the symmetry energy. Qualitatively thinking, the symmetry energy takes effect if the isospin fractionation mechanism is at work. The deeper the degree of isospin fractionation, the more sensitive the dependence of the probe on the symmetry energy probe. To check whether the isospin fractionation is relevant to the spacetime volume of the colliding system, the initial idea is to find a quantity that can be used to measure the degree of isospin fractionation. While the isobar model predicts the $\pi^{-} / \pi^{+}$ratio based on the static $N / Z$ composition of the system with $R_{\text {isob }}=\frac{5 N^{2}+N Z}{5 Z^{2}+N Z}$ [45], the transport model gives the $\pi^{-} / \pi^{+}$ratio $R_{\pi}$ based on the dynamic simulation of the compression (leading to high density) and the expansion (leading to low density) of the whole stage. Thus, to the first order of approximation, we adopt their difference $R_{\pi}-R_{\text {isob }}$ to represent the degree of isospin fractionation and plot it in Fig. 5 (a) as a function of the mass $A_{\text {sys }}$ for three systems with same $N / Z$ ratio, ${ }^{48} \mathrm{Ca}+{ }^{48} \mathrm{Ca}$, ${ }^{124} \mathrm{Sn}+{ }^{124} \mathrm{Sn}$ and ${ }^{197} \mathrm{Au}+{ }^{197} \mathrm{Au}$, at beam energies of 0.25 , 0.4 and $0.6 \mathrm{GeV} / \mathrm{u}$, respectively. The symmetry energy is parameterized with $x=1$ in the transport model calculation. It is seen that $R_{\pi}-R_{\text {isob }}$ exhibits a positive correlation to the system size at each beam energy, indicating a dependence of the isospin fractionation on the space-time volume of the colliding system. At lower beam energy, as the maximum density achieved is lower while the reaction time is longer, the net effect of the isovector potential on 

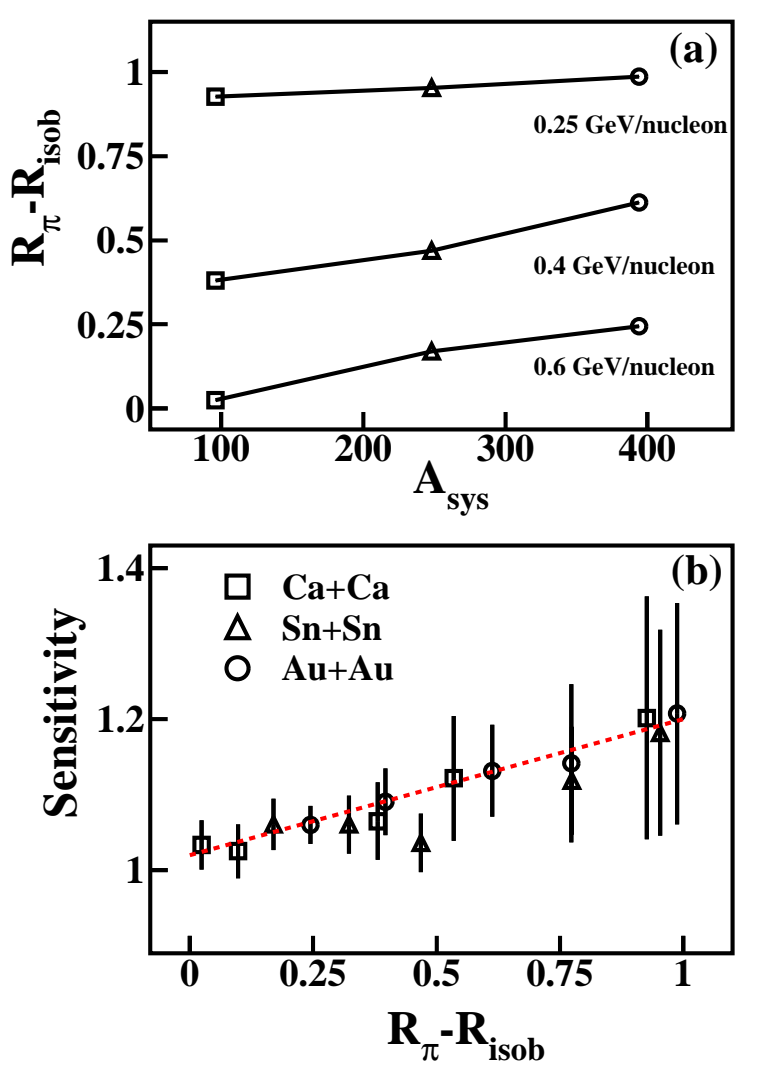

Fig. 5. (Color online) (a) the system size and beam energy dependence of the degree of isospin fractionation denoted by $R_{\pi}-R_{\text {isob }}$ (see text). (b) correlation between the sensitivity of the $\pi^{-} / \pi^{+}$ratio to the $E_{\text {sym }}(\rho)$ and the degree of isospin fractionation. The dashed line is for guiding the eyes. Taken from Ref. 55.

the $\pi^{-} / \pi^{+}$ratio is larger. At higher energies, besides the same trend, the $R_{\pi}-R_{\text {isob }}$ exhibit a more rapid increasing with $A_{\text {sys }}$ due to probably a larger isovector density gradients difference reached in the reactions. The sensitivity of the $\pi^{-} / \pi^{+}$ratio on the $E_{\text {sym }}(\rho)$, defined here by the double ratio of the $\pi^{-} / \pi^{+}$obtained with the $E_{\mathrm{sym}}(\rho)$ of $x=1$ over that with $x=0$, is plotted in Fig. 5(b) as a function of the $R_{\pi}-R_{\text {isob }}$. Interestingly it is shown that the sensitivity increases with the $R_{\pi}-R_{\text {isob }}$ despite of the larger uncertainty experienced at lower beam energies due to the low statistics of pions in the simulation. Thus it is suggested that the $\pi^{-} / \pi^{+}$ratio in heavy systems near the pion production threshold is preferred for probing the high densities behavior of the $E_{\text {sym }}(\rho)[55$.

It is of significance to clarify that the effect of the symmetry energy on the $\pi^{-} / \pi^{+}$ratio originates spatially from the high density region. M. Zhang et al. compares the effect of changing the $E_{\text {sym }}(\rho)$ parameter in high and low densities separately, suggesting that the high-density symmetry energy contributes about $80 \%$ of the total effect [55]. This conclusion is consistent with an earlier calcula-

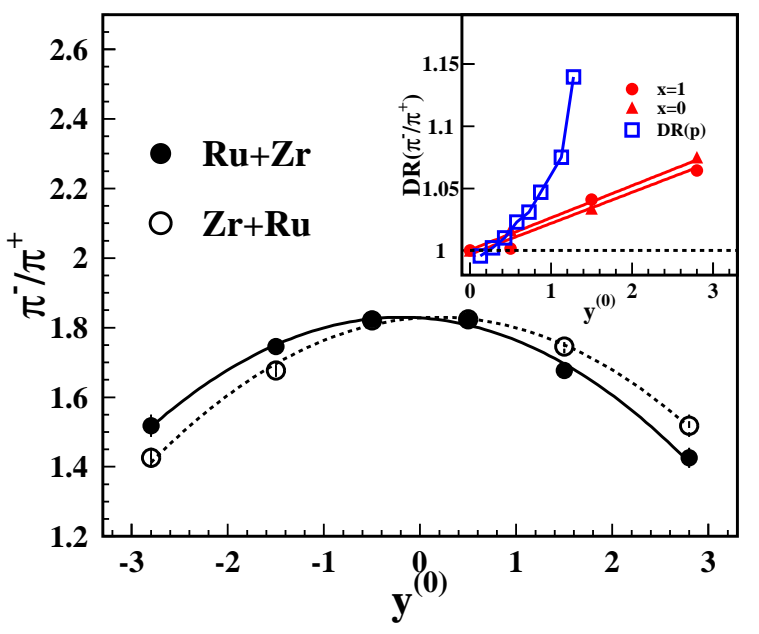

Fig. 6. (Color online) $\pi^{-} / \pi^{+}$ratio as a function of reduced rapidity $\left|y^{(0)}\right|$ in IBUU04 calculation with $x=1$ in ${ }^{96} \mathrm{Ru}+{ }^{96} \mathrm{Zr}$ (solid, calculated) and ${ }^{96} \mathrm{Zr}+{ }^{96} \mathrm{Ru}$ (open, reflected by the solid symbols with respect to the midrapidity). Taken from 56. The insert shows the double ratio $D R\left(\pi^{-} / \pi^{+}\right)$in the two systems with $x=0$ and 1 in comparison with the experimental ratio of proton $R(P)$ [57] (open square)

tion using URQMD transport model [8]. The isospin tracer with $\pi^{-} / \pi^{+}$as a function of rapidity may provide an indirect experimental test. According to transport simulation, the $\Delta$ resonances are mainly produced in high-density region, which is formed by the participants at earlier collision stage. Thus, in a simple picture, the pions are less influenced by the Corona effect than nucleons, for which the contribution from the surface is not negligible [56]. To see this effect, we study the pion ratio $\pi^{-} / \pi^{+}$in $\mathrm{Zr}+\mathrm{Ru}$ and $\mathrm{Ru}+\mathrm{Zr}$ collisions as a function of the reduced rapidity, as shown in Fig. 6. It is seen that a difference between the forward rapidity and backward rapidity is evident, suggesting the isospin equilibrium is not achieved. More interestingly one can see from the insert of Fig. 6 that the double ratio of charged pions $\operatorname{DR}\left(\pi^{-} / \pi^{+}\right)$in $\mathrm{Ru}+\mathrm{Zr}$ and $\mathrm{Zr}+\mathrm{Ru}$ systems shows approximately linear increase with rapidity while the ratio of proton in these two systems exhibits a more rapid increase with rapidity. The different behavior between the pions and the protons suggests that the surface effect strongly experienced by the later is less pronounced for the pions. If this effect is confirmed by the experiment, it suggests that the primordial pions are mainly produced in the high-density region, which is in agreement with earlier transport model calculations [55].

\subsection{The $K^{+} / K^{0}$ ratio as a probe to nuclear symmetry energy}

Compared with pions, kaons are also produced in the dense phase in heavy-ion collisions but experience less final state interactions and thus provide a useful probe of 
the EOS of nuclear matter at high densities 58. Earlier, the kaon yield has been used successfully in constraining the incompressibility of isospin symmetric nuclear matter. For instance, transport model analyses of the Kaos data suggest that the EOS of symmetric matter is soft 59,60,61. For the isospin effect, Q. Li et al has noticed in ${ }^{132} \mathrm{Sn}+{ }^{132} \mathrm{Sn}$ at $1.5 \mathrm{GeV} / \mathrm{u}$ that the $K^{+} / K^{0}$ ratio depends on the symmetry potential 8. Later in 9, an excitation function of $K^{+} / K^{0}$ in $\mathrm{Au}+\mathrm{Au}$ collisions with different symmetry potential calculated and confirmed the dependence of the $K^{+} / K^{0}$ ratio on the isovector potential. It has been realized that the isovector part of the inmedium interaction affects the kaon production via two mechanisms: (i) a symmetry potential effect, i.e., a larger neutron repulsion in n-rich systems, and (ii) a threshold effect, due to the change in the self-energies of the particles involved in inelastic processes. Thus the $K^{+} / K^{0}$ ratio was proposed as another, probably better, probe of the highdensity behavior of nuclear symmetry energy 9. Similar to the $\pi^{-} / \pi^{+}$observable, the sensitivity of the $K^{+} / K^{0}$ to the variation of symmetry energy is more pronounced at beam energies near the kaon production threshold [8,9].

The first attempt to extract the stiffness of symmetry energy from $K^{+} / K^{0}$ observable was done by the FOPI collaboration 62 . To reduce the systematic uncertainties, the double ratio of $K^{+} / K^{0}$ in $\mathrm{Zr}+\mathrm{Zr}$ and $\mathrm{Ru}+\mathrm{Ru}$ systems with same mass but characterized by different $N / Z$ composition at $1.528 \mathrm{GeV} / \mathrm{u}$ is used. The experimental result is compared with thermal model calculation and a relativistic mean field transport model using two different collision scenarios (the infinite nuclear matter and the realistic finite nuclear collision) and under different assumptions on the stiffness of the symmetry energy, as presented in Fig. 7. Thermal model calculation reproduces the data. For the transport model calculations, the sensitivity of the double ratio $K^{+} / K^{0}$ on the stiffness of symmetry energy differs in the two scenarios. In the former case, it is evident that the double ratio $K^{+} / K^{0}$ increases rapidly if one goes from $N L$ (symmetry energy only containing kinetic contribution) via $N L \rho$ (including the isovector-vector field $\rho$ ) to $N L \rho \delta$ (including both $\rho$ and $\delta$ effective field), or equivalently with increasing the stiffness of $E_{\text {sym }}(\rho)$ from 50 to $100 \mathrm{MeV}$ at $2.5 \rho_{0}$. While in the realistic nuclear collisions of $\mathrm{Ru} / \mathrm{Zr}$ nuclei, the sensitivity is less pronounced and the double ratio of $K^{+} / K^{0}$ differs by about $5 \%$ between $N L$ and $N L \rho \delta$ parameterizations. This reduced sensitivity is mainly attributed to two dynamical effects that tend to weaken the contribution of the isovector interaction, i.e., the fast neutron emission and the transformation of neutron into proton in inelastic channels. It indicates that to obtain experimentally accessible sensitivity, one shall use the lower beam energy in heavier system with larger $N / Z$ asymmetry. On the experimental side, however, measuring a sample of $K^{0}$ and $K^{+}$with sufficiently high statistics and high precision at subthreshold energies will be a considerable challenge and calls for great effort in future experiment.

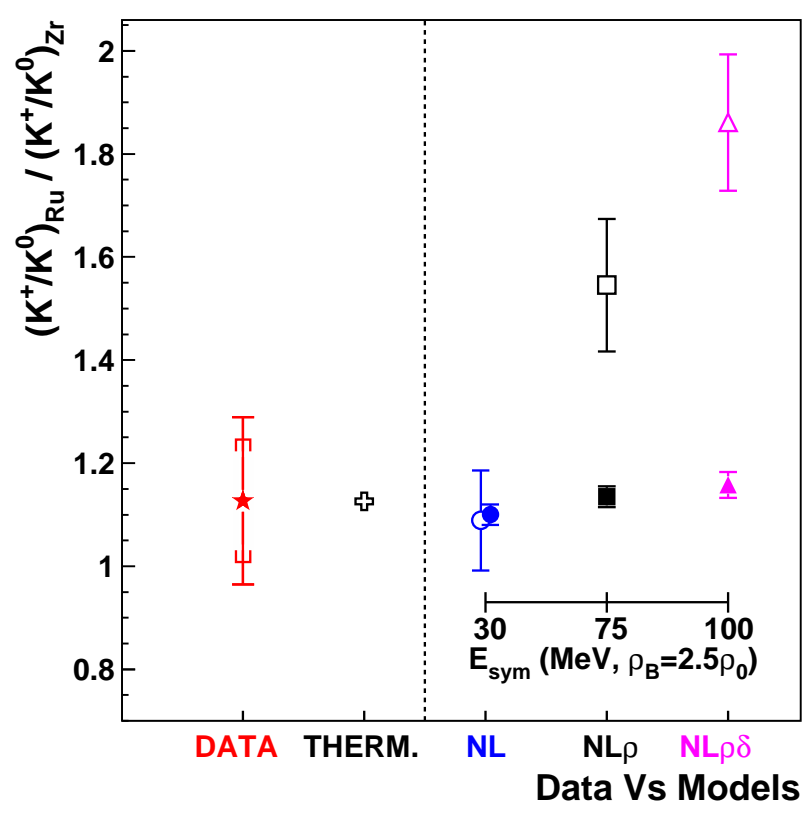

Fig. 7. (Color Online) The double ratio of $K^{+} / K^{0}$ measured in $\mathrm{Ru}+\mathrm{Ru}$ and $\mathrm{Zr}+\mathrm{Zr}$ systems (red star) in comparison with thermal model calculations (open cross in left panel) and relativistic mean field transport model (right panel) for infinite nuclear system (open) and realistic collisions (solid), taken from Ref. 62.

\section{3 $\eta$ production and effects of nuclear symmetry energy}

This subsection is a brief summary of the work originally published in ref. 16. Given the inconsisteny of the high density $E_{\text {sym }}(\rho)$ among various transport model analyses, searching for more sensitive new probes of $E_{\text {sym }}(\rho)$ at high-densities is still ongoing. Compared to pion or kaon, massive mesons generally probe the $E_{\text {sym }}(\rho)$ at higher densities if the effects of the final state interactions do not smear the signal. The $\eta$ meson, as the massive member in the nonet of pseudoscalar Goldstone mesons with mass $547.853 \mathrm{MeV} / \mathrm{c}^{2} 63$, is a preferred probe to $E_{\mathrm{sym}}(\rho)$ for further good reasons: (1) The $\eta$ meson experiences weaker final state interactions compared to pion due to its hidden strangeness (the $s \bar{s}$ component). And because the net strangeness content is zero in $\eta$ mesons, they can be produced without another strange partner in the final state and thus require less energies. In addition, it has significant photon and dilepton decay branch ratio, which provides a clean electromagnetic probe to $E_{\text {sym }}(\rho)$. (2) The $\eta$ meson is sensitive to the number of $\mathrm{p}-\mathrm{n}$ collisions while the $\pi^{-} / \pi^{+}$ratio is determined by the ratio of $n-n$ and $p-p$ colliding pairs, $\eta$ and the $\pi^{-} / \pi^{+}$ratio provide complementary information about the symmetry energy. (3) The $\eta$ meson is produced from the proton-neutron collision for which the relative momentum is determined mainly by the gradient of the isovector potential. However, we shall keep in mind that the elementary $\eta$ production cross sections 


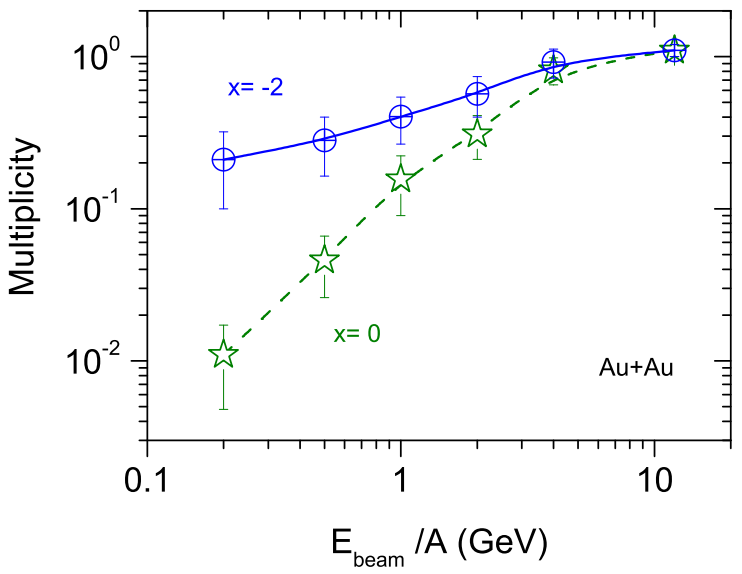

Fig. 8. (Color online) Effects of the symmetry energy on the multiplicity of inclusive $\eta$ production as a function of incident beam energy in $\mathrm{Au}+\mathrm{Au}$ reactions using a relativistic transport model ART1.0. Figure is taken from Ref. [16.

in baryon-baryon and meson-baryon scattering still suffer from larger uncertainties although they are gradually better known as more data and calculations become available 64,65. Moreover, the detection of the photonic decay or Dalitz decay (with dilepton) of $\eta$ is also challenging near the production threshold.

The sub-threshold $\eta$ production requires multiple nucleo nucleon scattering over sufficiently long time 66 and hence carries mainly the information of the equilibrium phase. Fig. 8 presents the $\eta$ multiplicity as a function of beam energy in inclusive $\mathrm{Au}+\mathrm{Au}$ reactions with both soft $(x=$ $0)$ and stiff $(x=-2)$ symmetry energies using a relativistic transport model ART1.0 67. One can clearly see that the $\eta$ multiplicity decreases rapidly with decreasing the beam energy especially for the soft symmetry energy but saturates at about $10 \mathrm{GeV} / \mathrm{u}$ with increasing the beam energy. Over the whole energy range under investigation, more (less) $\eta$ mesons are produced with the stiff (soft) symmetry energy. Again due to the isospin fractionation mechanism in the equilibrium phase, the symmetry energy and the isospin asymmetry $\delta$ satisfies the equation $E_{\text {sym }}\left(\rho_{1}\right) \delta_{1}=E_{\text {sym }}\left(\rho_{2}\right) \delta_{2}$ where $\rho_{1}$ and $\rho_{2}$ represents two density conditions, respectively 68, 35. Thus, with a higher symmetry energy $E_{\text {sym }}(\rho)$ the dense region is less isospin asymmetric and more $n p(p n)$ collisions can occur to produce more $\eta$ mesons. Interestingly, the effect of the nuclear symmetry energy becomes stronger and stronger with decreasing beam energy and the sensitivity of the $\eta$ production on $E_{\text {sym }}(\rho)$ is more pronounced in comparison to the probe of $\pi^{-} / \pi^{+}$ratio in the same energy region.

Fig. 9 further shows the transverse momentum distribution of $\eta$ in $\mathrm{Au}+\mathrm{Au}$ reactions at beam energy of 200 $\mathrm{MeV} / \mathrm{u}$ with different density-dependent symmetry energies and incompressibility coefficients $K$. The ordinate $d M_{\eta} / d p_{t}$ represents the differential $\eta$ multiplicity defined

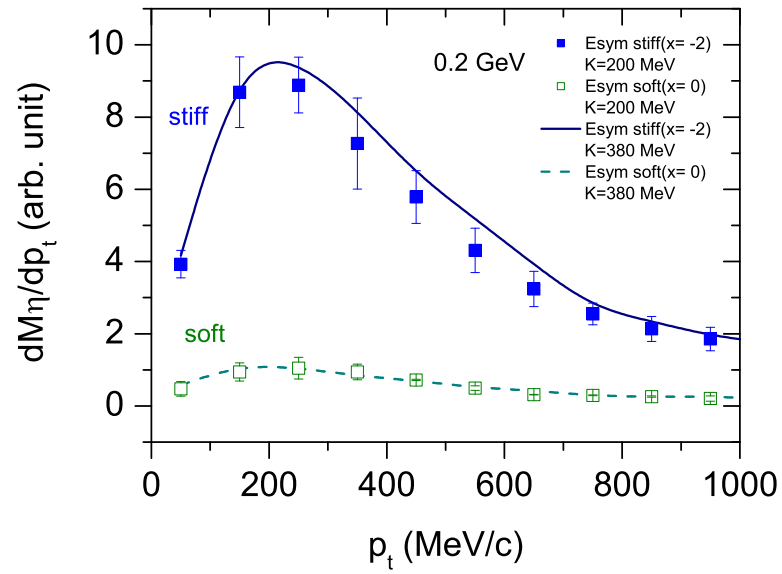

Fig. 9. (Color online) Effects of the symmetry energy and the incompressibility of symmetric nuclear matter on the transverse momentum distributions of $\eta$ production in inclusive $\mathrm{Au}+\mathrm{Au}$ reaction at incident beam energy of $200 \mathrm{MeV} / \mathrm{u}$ using a relativistic transport model ART1.0. Figure is taken from Ref. [16].

by $\frac{M_{\eta}\left(p_{t} \rightarrow p_{t}+d p_{t}\right)}{d p_{t}}$. By comparing the two curves it is seen that the effect of $E_{\mathrm{sym}}(\rho)$ is most pronounced in the $p_{t}$ interval from about 200 to $300 \mathrm{MeV} / \mathrm{c}$. The predictions with two $E_{\text {sym }}(\rho)$ parameters of $x=0$ and -2 differs by a factor of about 5 to 10 , while the effect of changing the incompressibility of nuclear matter from $K=200$ $\mathrm{MeV}$ to $380 \mathrm{MeV}$ is insignificant. This is attributed to the fact that the $p-n$ relative momentum is essentially determined by the gradient of the isovector potential. For the isoscalar part, the effects of the incompressibility $K$ of the symmetric nuclear matter on the production and the reabsorption of $\eta$ nearly cancel out. It can be understood that a large $K$ causes less resonances (and hence less $\eta$ ) to be produced, on the other hand a hard symmetric matter undergoes a fast expansion causing less reabsorption. The effect of utilizing a momentum dependent isoscalar potential is worthy of further investigation.

\subsection{High energy photon production and effects of nuclear symmetry energy}

This subsection is a brief summary of the work originally published in ref. [17. The hadronic probes of the nuclear symmetry energy usually suffer from strong final state interactions (FSI), although some special treatment, such as using the squeezed-out neutron/proton ratio perpendicular to the reaction plane 12, tends to reduce strong FSI effect. To overcome this difficulty, the electromagnetic observables, such as the photons or dileptons, are proposed as cleaner probes to the nuclear symmetry energy $E_{\text {sym }}(\rho)$, especially at supranormal densities.

During the last twenty years, hard photon emissions in heavy ion reactions at various beam energies have been 


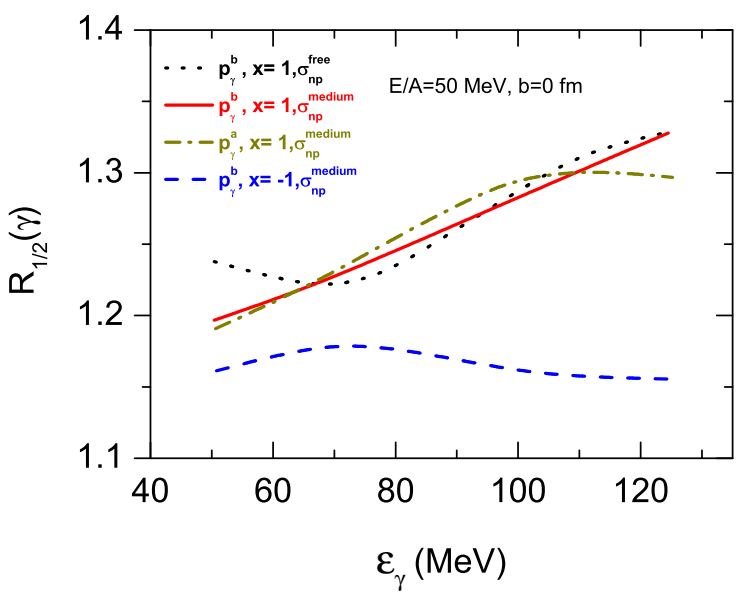

Fig. 10. (Color online) The spectra ratio of hard photons in the reactions of ${ }^{132} S n+{ }^{124} S n$ and ${ }^{112} S n+{ }^{112} S n$ reactions at a beam energy of $50 \mathrm{MeV} / \mathrm{u}$ using IBUU04 transport model with interaction $\operatorname{MDI}(x=-1)$ (very stiff symmetry energy) and $\operatorname{MDI}(x=1)$ (very soft symmetry energy), respectively. Taken from Ref. [17.

extensively studied both theoretically and experimentally by several groups 69,70,71. It has been concluded that the high energy $\gamma$ rays are mainly produced in the early stage of the reaction and have very low probability to experience final state interaction. Thus besides the symmetry energy effect, the emitting hard photons can probe not only the reaction dynamics leading to the formation of dense matter $[72,73,74,75,76$, but also the in-medium NN (nucleon-nucleon) scattering cross sections [77. Nevertheless, the major uncertainty of the probability $p_{\gamma}$ of the input elementary process $p n \rightarrow p n \gamma$, which is a major concern so far, is still rather model dependent $78,79,80$. 81, 82.

Since a double ratio of a certain observable in two reactions usually reduce the systematic uncertainty and other "unwanted effects", as early discussed in Ref. [17, we have proposed to measure the spectra ratio $R_{1 / 2}(\gamma)$ of hard photons from two head-on reactions of ${ }^{132} \mathrm{Sn}+{ }^{124} \mathrm{Sn}$ and ${ }^{112} \mathrm{Sn}+{ }^{112} \mathrm{Sn}$, i.e.,

$$
R_{1 / 2}(\gamma) \equiv \frac{\frac{d N}{d \varepsilon_{\gamma}}\left({ }^{132} \mathrm{Sn}+{ }^{124} \mathrm{Sn}\right)}{\frac{d N}{d \varepsilon_{\gamma}}\left({ }^{112} \mathrm{Sn}+{ }^{112} \mathrm{Sn}\right)}
$$

By choosing the two systems with approximately equal mass but rather different isospin compositions, we hope to see the isospin effect. Shown in Fig. 10 is the $R_{1 / 2}(\gamma)$ calculated using IBUU04 transport model with different symmetry energy stiffnesses, i.e., $\operatorname{MDI}(x=-1)$ (very stiff) and $\operatorname{MDI}(x=1)$ (very soft), respectively. To show the effects arising from the two body scattering, the process probability of the elementary process $p n \rightarrow p n \gamma$ is taken in two forms, $p_{\gamma}^{a}$ and $p_{\gamma}^{b}$ [17] and the NN cross section is also varied in the calculation with $\sigma_{\text {np }}^{\text {free being the cross }}$ section in free space and $\sigma_{\mathrm{np}}^{\text {medium }}$ being the value modified due to medium effects, respectively. As expected, the full calculations with the $p_{\gamma}^{a}$ and $p_{\gamma}^{b}$ and different NN cross sections indeed lead to about the same $R_{1 / 2}(\gamma)$ within statistical uncertainties since the corresponding effects are canceled out by the ratio method. Comparing the results with $(x=1)$ and $\operatorname{MDI}(x=-1)$ both using the $p_{\gamma}^{b}$, however, it is clearly seen that the $R_{1 / 2}(\gamma)$ remains sensitive to the symmetry energy especially for the very energetic photons. This verify numerically the advantage of using the $R_{1 / 2}(\gamma)$ as a robust probe of the symmetry energy. Despite of the significant effect, we would also note here that the detection of hard photons is also challenging and requires careful treatment since the $\gamma$ rays from other processes are involved in heavy ion collisions in the relevant energy range.

\section{Perspective}

In order to have insight into the nuclear equation of state at supra-saturation densities using heavy-ion collisions, it is a prerequisite that a compressed phase of nuclear matter, which is the environment where the $E_{\text {sym }}(\rho)$ takes effect, can be formed and last for adequately long time. In the collisions, not only the symmetry potential as a part of the mean field, but also many other factors like the inmedium modification of the hadrons properties and the two body collision cross sections come into play. In addition, the compression, expansion and cooling processes are dynamically correlated in heavy-ion collisions. Transport models providing consistent description of the multi facets of the collisions are required to bridge the nuclear equation of state and the experimental observables in order to draw model- and observable- independent conclusions on the $E_{\text {sym }}(\rho)$.

From experimental side, we have shown that heavyion collisions in sub- $\mathrm{GeV} / \mathrm{u}$ regime is beneficial for the experimental constraining of symmetry energy in terrestrial laboratory. Despite of many experimental challenges, some worldwide radioactive beam facilities and the experiments below $1 \mathrm{GeV} / \mathrm{u}$ bombarding energy and with larger beam intensity are under constructions. For instance, the ASY-EOS experiment at GSI in Germany is dedicated to measure the differential neutron and proton flow in heavy ion collisions at several hundreds $\mathrm{MeV} / \mathrm{u} 83,84,85$. The SAMURAI spectrometer has been constructed at RIKEN in Japan [86, which was designed for kinematically complete radioactive beam experiments, including the pion production measurement in near threshold heavy ion collisions. The large-acceptance multipurpose spectrometer (LAMPS) is to be constructed on RAON in Korean 87. The high energy stage of LAMPS combines a solenoid and a dipole with neutron detectors. It will be able to measure charged pions, fragments and neutrons with large coverage and hence provides further experimental data in constraining the high density symmetry energy. Besides, the cooling storage ring (HIRFL-CSR) in China, delivering heavy-ion beams up to $1 \mathrm{GeV} / \mathrm{u}[88$, to be coupled with 
advanced detectors at the external target experiment as called CEE (CSR External-target Experiment), will also contribute to further studies of $E_{\text {sym }}(\rho)$ 89. The central components of CEE are the tracking detectors inside and downstream to a dipole with large acceptance at forward and midrapidity in laboratory reference. For the forward rapidity acceptance, it is expected to provide complementary data to the existing devices [90. Meanwhile, further observables, for instance, the balance energy of direct nucleon flow 91, the scattering angles of the nucleons on a heavy target 92, are demonstrated sensitively probing the symmetry energy at high densities. With the running of these experiments and the cross check with more observables coming into play, the next decade will hopefully witness more abundant data leading to further constraints of the high-density behavior of $E_{\mathrm{sym}}(\rho)$.

\section{Summary}

Some recent progress and remaining issues in constraining the symmetry energy at supra-saturation densities in heavy-ion collisions are reviewed. Large uncertainties of the behavior of the symmetry energy beyond saturation density still exist both theoretically and experimentally. Based on BUU and Boltzmann-Langevin transport model analysis of the most recent systematic pion data, circumstantial evidence suggesting a rather soft symmetry energy is obtained. However, this conclusion is different from those based on some other model analyses. Further simulations suggest that large symmetry energy sensitivity of the charged pion ratio can be expected in heavy-ion collisions below $0.5 \mathrm{GeV} / \mathrm{u}$, supporting further studies at several new facilities. After being proposed as a promising probe, Kaonic ratio $K^{+} / K^{0}$ has been measured in $\mathrm{Ru} / \mathrm{Zr}$ collisions at $1.58 \mathrm{GeV} / \mathrm{u}$ but does not lead to a convincing constraint of $E_{\text {sym }}(\rho)$ because the symmetry energy effect can not be discriminated within the current experimental uncertainty. With the discrepancy of the high-density behavior of the symmetry energy remaining unresolved, more sensitive probes of relevance are always useful. $\eta$ and photon production are also potential tools to constrain the symmetry energy. Further experimental studies and theoretic work are required.

\section{Acknowledgement}

This work is supported by the CUSTIPEN (China-U.S. Theory Institute for Physics with Exotic Nuclei) under DOE grant number DE-FG02-13ER42025, the US National Science Foundation under Grant No. PHY-1068022, the National Aeronautics and Space Administration under grant NNX11AC41G issued through the Science Mission Directorate, the Natural Science Foundation of China under Grant No. 11079025, 11135011, 11275125, 11375239, 11375094 and U1332207 Tsinghua University Initiative Scientific Research Program, the Shanghai Rising-Star Program under grant number 11QH1401100, the "Shu Guang" project supported by Shanghai Municipal Education Commission and Shanghai Education Development Foundation, the Program for Professor of Special Appointment (Eastern Scholar) at Shanghai Institutions of Higher Learning, and the Science and Technology Commission of Shanghai Municipality (11DZ2260700).

\section{References}

1. B.A. Li, L.W. Chen, and C.M. Ko, Phys. Rep. 464 (2008) 113

2. B.A. Li et al., Journal of Physics: Conference Series 312 (2011) 042006

3. B. Tsang et al., Phys. Rev. C86 (2012) 015803

4. J.M. Lattimer, Ann. Rev. Nucl. Part. Sci. 62 (2012) 485

5. L.W. Chen, Proceedings of the 14th National Conference on Nuclear Structure in China (NSC2012) (World Scientific, Singapore, 2012), pp. 43-54 arXiv:1212.0284.

6. B.A. Li et al., J. Phys.: Conf. Series 413 (2013) 012021 arXiv:1212.1178.

7. B.A. Li et al., Phys. Rev. C71 (2005) 014608

8. Q. Li, Z. Li et al., J. Phys. G: Nucl. Part. Phys. 31 (2005) 1359

9. G. Ferini et al., Phys. Rev. Lett 97 (2006) 202301

10. B.A. Li, Phys. Rev. Lett. 88 (2002) 192701

11. P. Russotto, et al., Physics Letters B 697, 471 (2011)

12. G.C. Yong, B.A. Li and L.W. Chen, Phys. Lett. B650 (2007) 344

13. L.W. Chen, C.M. Ki, and B.A. Li, Nucl. Phys. A729 (2003) 809

14. G.C. Yong, B.A. Li, L.W. Chen, and X.C. Zhang, Phys. Rev. C80 (2009) 044608

15. Q. Li, Z. Li, E. Zhao, and R.K. Gupta, Phys. Rev. C71 (2005) 054907

16. G.C. Yong and B.A Li, Phys. Let. B723 (2013) 388.

17. G.C. Yong, B.A. Li and L.W. Chen, Phys. Lett. B661 (2008) 82.

18. Y.G. Ma et al., Phys. Rev. C85 (2012) 024618

19. W. Reisdorf, et al. for FOPI collaboration, Nucl. Phys. A781 (2007) 459

20. B.A. Li, C.B. Das, S. Das Gupta and C. Gale, Phys. Rev. C69 (2004) 011603; Nucl. Phys. A735 (2004) 563.

21. Z.G. Xiao et al., Phys. Rev. Lett. 102 (2009) 062502

22. Z.G. Xiao et al., Sci. Sin. Phys. Mech. Astron. 41, 439 (2011)

23. Z.Q. Feng and G. M. Jin, Physics Letters B683 (2010) 140

24. W.J. Xie et al, Physics Letters B718 (2013) 1510

25. J. Xu, C.M. Ko, and Y. Oh, Phys. Rev. C81 (2010) 024910

26. G.C. Yong, The European Physical Journal A46 (2010) 399

27. J. Xu, L.W. Chen, C.K. Ko, B.A. Li, and Y.G. Ma, Phys. Rev. C87 (2013) 067601.

28. C. Xu, A. Li and B.A. Li, Phys. Rev. C81 (2010) 064612.

29. W. Zuo and U. Lombardo, EPJA, this issue.

30. C. Xu, A. Li and B.A. Li, J. of Physics: Conference Series 420 (2013) 012190.

31. H.K. Lee and Mannque Rho, EPJA this issue.

32. L.W. Chen et al., Phys. Rev. C80 (2009) 014322

33. L.W. Chen, Science China: Physics, Mechanics and Astronomy 54 (2011) s124 arXiv:1101.2384.

34. W.Z. Jiang and B.A. Li Phys. Rev. C80 (2009) 044322 
35. B.A. Li, Nucl. Phys. A708 (2002) 365.

36. H. Müller and B. D. Serot, Phys. Rev. C 52 (1995) 2072.

37. B.A. Li and C. M. Ko, Nucl. Phys. A618, (1997) 498.

38. H.S. Xu et al., Phys. Rev. Lett. 85 (2000) 716

39. B.A. Li et al., Phys. Rev. C67 (2003) 017601

40. G. Ferini, M. Colonna, T. Gaitanos and M. Di Toro, Nucl.

Phys. A762, (2005) 147.

41. W. Benenson et al., Phys. Rev. Lett. 43, 683 (1979).

42. M. D. Cozma, Phys. Lett. B700 (2011) 139.

43. S. Nagamiya et al., Phys. Rev. C24, 971 (1981).

44. J. Harris et al., Phys. Lett. B153, 377 (1985).

45. R. Stock, Phys. Rep., 135, 259 (1986).

46. G.F. Bertsch, Nature 283, 280 (1980).

47. H.R. Jaqaman, A.Z. Mekjian and L. Zamick, Phys. Rev. C27, 2782 (1983); ibid, C29, 2067 (1984); H.R. Jaqaman, Phys. Rev. C39, 169 (1988).

48. C. Xu, B. A. Li, and L. W. Chen, Phys. Rev. C 82 (2010) 054607

49. C. Xu, B. A. Li, L. W. Chen, and C. M. Ko, Nucl. Phys. A 865 (2011) 1

50. R. Chen, B.J. Cai, L.W. Chen, B.A. Li, X.H. Li, and C. Xu, Phys. Rev. C 85 (2012) 024305

51. L.W. Chen, C.M. Ko, and B.A. Li, Phys. Rev. Lett. 94 (2005) 032701

52. A. Akmal et al., Phys. Rev. C58, 1804 (1998).

53. C. Hartnack et al., Eur. Phys. J. A1 151 (1998) 1

54. D.H. Wen et al., Phys. Rev. Lett 103 (2009) 211102

55. M. Zhang et al., Phys. Rev. C80 (2009) 034616

56. M. Zhang et al., Phys. Rev. C82 (2010) 044602

57. B. Hong and FOPI collaboration, Phys. Rev. C 66 (2002) 034901

58. J. Aichelin and C.M. Ko, Phys. Rev. Lett. 55 (1985) 2661.

59. C. Sturm et al. for KaoS collaboration, Phys. Rev. Lett. 86 (2001) 39

60. Ch. Hartnack, H. Oeschler et al., Phys. Rev. Lett. 96 (2006) 012302

61. C. Fuchs et al., Phys. Rev. Lett 86(2001) 1974

62. X. Lopez, Y. J. Kim et al, Phys. Rev. C75 (2007) 011901(R)

63. J. Beringer et al. (Particle Data Group), Phys. Rev. D86 (2012) 010001.

64. Gy. Wolf, W. Cassing and U. Mosel, Nucl. Phys. A552 (1993) 549.

65. M. Thomere, C. Hartnack, G. Wolf and J. Aichelin, Phys. Rev. C75 (2007) 064902.

66. A. Depaoli, K. Niita, W. Cassing, U. Mosel and C.M. Ko, Phys. Lett. B219 (1989) 194.

67. B.A. Li and C.M. Ko, Phys. Rev. C 52 (1995) 2037.

68. L. Shi and P. Danielewicz, Europhys. Lett. 49 (2000) 34.

69. G.F. Bertsch and S. Das Gupta, Phys. Rep. 160189 (1988).

70. H. Nifenecker and J.A. Pinston, Annu. Rev. Nucl. Part. Sci. 1990. 40: 113-43.

71. W. Cassing, V. Metag, U. Mosel, and K. Niita, Phys. Rep. 188 (1990) 363.

72. B. A. Remington, M. Blann and G.F. Bertsch, Phys. Rev. Lett. 57 (1986) 2909.

73. C.M. Ko, G.F. Bertsch and J. Aichelin, Phys. Rev. C31 (1985) 2324(R).

74. W. Cassing, T. Biro, U. Mosel, M. Tohyama, and W. Bauer, Phys. Lett. B181 (1986) 217.

75. W. Bauer, G.F. Bertsch, W. Cassing and U. Mosel, Phys. Rev. C34 (1986) 2127.
76. J. Stevenson et al., Phys. Rev. Lett. 57 (1986) 555.

77. G.C. Yong, W. Zuo and X.C. Zhang, Phys. Lett. B705 (2011) 240.

78. H. Nifenecker and J.P. Bondorf, Nucl. Phys. A442 (1985) 478.

79. K. Nakayama and G.F. Bertsch, Phys. Rev. C34 2190 (1986).

80. M. Schäffer, T.S. Biro, W. Cassing and U. Mosel, H. Nifenecker and J.A. Pinstan, Z. Phys. A339 391 (1991).

81. N. Gan et al., Phys. Rev. C49 (1994) 298.

82. R.G.E. Timmermans, T.D. Penninga, B.F. Gibson, M.K. Liou, Phys. Rev. C73 (2006) 034006.

83. P. Russotto et al., J. Phys.: Conf. Ser. 420 (2013) 012092

84. P. Russotto, M.D. Cozma et al., The European Physical Journal A, this issue.

85. W. Trautmann et al., The European Physical Journal A, this issue.

86. T. Kobayashi et al, Nucl. Inst. Meth. 2013, accepted. DOI: http://dx.doi.org/10.1016/j.nimb.2013.05.089

87. B. Hong et al, The European Physical Journal A, this issue.

88. J.W. Xia et al, Nucl. Instr. Meth. A488 (2002) 11

89. Z.G. Xiao et al, J. Phys. G: Nucl. Part. Phys. 36 (2009) 064040

90. M. Zhang et al, Chin. Phys. C34 (2010) 1100

91. C. C. Guo et al., Science China A 55 (2012) 252

92. L. Ou et al., Phys. Rev. C78 (2008) 044609. 